\title{
Spin dynamics in relativistic light-matter interaction
}

\author{
Heiko Bauke, ${ }^{1}$, Sven Ahrens, ${ }^{1,2, f^{7}}$ Christoph H. Keitel, ${ }^{1}$ and Rainer Grobe ${ }^{1,2}$ \\ ${ }^{1}$ Max-Planck-Institut für Kernphysik, Saupfercheckweg 1, 69117 Heidelberg, Germany \\ ${ }^{2}$ Intense Laser Physics Theory Unit and Department of Physics, \\ Illinois State University, Normal, Illinois 61790-4560 USA
}

\begin{abstract}
Various spin effects are expected to become observable in light-matter interaction at relativistic intensities. Relativistic quantum mechanics equipped with a suitable relativistic spin operator forms the theoretical foundation for describing these effects. Various proposals for relativistic spin operators have been offered by different authors, which are presented in a unified way. As a result of the operators' mathematical properties only the FoldyWouthuysen operator and the Pryce operator qualify as possible proper relativistic spin operators. The ground states of highly charged hydrogen-like ions can be utilized to identify a legitimate relativistic spin operator experimentally. Subsequently, the Foldy-Wouthuysen spin operator is employed to study electron-spin precession in high-intensity standing light waves with elliptical polarization. For a correct theoretical description of the predicted electron-spin precession relativistic effects due to the spin angular momentum of the electromagnetic wave has to be taken into account even in the limit of low intensities.
\end{abstract}

\section{Introduction}

Employing novel light sources such as the ELI-Ultra High Field Facility, for example, that envisage to provide field intensities in excess of $10^{20} \mathrm{~W} / \mathrm{cm}^{2}$ and field frequencies in the x-ray domain [1-5] light-matter interaction in the relativistic regime may be probed experimentally. Relativistic quantum mechanics predicts various new phenomena to occur in this regime [6, 7], for example, multiphoton scattering, radiation reaction effects, vacuum-polarization effects or even pair creation [8-10]. Furthermore, electrons in strong electromagnetic fields can exhibit distinct spin effects [11-16].

Relativistic quantum mechanics has to be employed to study spin phenomena in strong electromagnetic fields. According to the formalism of quantum mechanics, each measurable quantity - as the spin, for example - is represented by a Hermitian operator. However, there is no universally accepted operator to describe an electron's spin degree of freedom within the framework of relativistic quantum mechanics. Thus, we first investigate the properties of different proposals for a relativistic spin operator and show that most candidates are lacking essential features of proper angular momentum operators [17, 18]. Only the so-called Foldy-Wouthuysen and the Pryce operators qualify as proper relativistic spin operators. The various spin operators predict different expectation values when electrons interact with electromagnetic potentials. In this way, one may distinguish between the proposed relativistic spin operators by experimental means. In particular, eigenstates of highly charged hydrogen-like ions may be utilized to identify a legitimate relativistic spin operator experimentally.

A further relativistic spin phenomenon, which we study in more detail, is the coupling of the spin angular momentum of light beams with elliptical polarization to the spin degree of freedom of free electrons [19, 20]. This coupling, which is of similar origin as the well-known spin-orbit coupling, and the magnetic field lead to electron-spin precession. The spin-precession frequency is proportional to the product of the laser-field's intensity and its spin density. To derive the correct spin-precession frequency relativistic corrections to the nonrelativistic Pauli equation, which account for the light's spin density, have to be taken into account. The quantum mechanical interactions of the electron's spin with the laser's rotating magnetic field, which may be characterized by the nonrelativistic Pauli equation, and the electron spin's interaction with the laser field's spin density, which results via the relativistic corrections, counteract each other. As a result, a net electron-spin rotation remains with a precession frequency that is much smaller than the frequency predicted by a nonrelativistic theory. These relativistic effects are maintained even if the involved electromagnetic field strengths are nonrelativistic.

\section{Relativistic spin operators}

A Lorentz invariant quantum mechanical description of the motion of an electron in electromagnetic fields is provided by the time-dependent Dirac equation. For a particle of rest mass $m_{0}$ and charge $q$ it is given by (units are used in this section for which

\footnotetext{
*heiko.bauke@mpi-hd.mpg.de

$\dagger$ Current affiliation: Beijing Computational Science Research Center, Beijing 100094, China
} 
$\hbar=1)$

$$
\mathrm{i} \frac{\partial \Psi(\boldsymbol{r}, t)}{\partial t}=\hat{H} \Psi(\boldsymbol{r}, t)=\left(c \boldsymbol{\alpha} \cdot(\hat{\boldsymbol{p}}-q \boldsymbol{A}(\boldsymbol{r}, t))+q \phi(\boldsymbol{r}, t)+m_{0} c^{2} \beta\right) \Psi(\boldsymbol{r}, t),
$$

with the electromagnetic potentials $\phi(\boldsymbol{r}, t)$ and $\boldsymbol{A}(\boldsymbol{r}, t)$, the speed of light $c$, the canonical momentum operator $\hat{\boldsymbol{p}}=-\mathrm{i} \boldsymbol{\nabla}$, and the matrices $\boldsymbol{\alpha}=\left(\alpha_{1}, \alpha_{2}, \alpha_{3}\right)^{\mathrm{T}}$ and $\beta$. These $4 \times 4$ matrices obey the algebra

$$
\alpha_{i}^{2}=\beta^{2}=1, \quad \alpha_{i} \alpha_{k}+\alpha_{k} \alpha_{i}=2 \delta_{i, k}, \quad \alpha_{i} \beta+\beta \alpha_{i}=0 .
$$

To specify our notation and abbreviations, we employ the Dirac representation, where the matrices $\alpha_{i}$ and $\beta$ are defined as

$$
\alpha_{i}=\left(\begin{array}{cc}
0 & \sigma_{i} \\
\sigma_{i} & 0
\end{array}\right) \quad \text { for } i=1,2,3, \quad \beta=\left(\begin{array}{cc}
\mathbb{I}_{2} & 0 \\
0 & -\mathbb{I}_{2}
\end{array}\right)
$$

in terms of the three $2 \times 2$ Pauli matrices $\sigma=\left(\sigma_{1}, \sigma_{2}, \sigma_{3}\right)^{\top}$. In the Dirac representation, the Pauli matrices are given by

$$
\sigma_{1}=\left(\begin{array}{cc}
0 & 1 \\
1 & 0
\end{array}\right), \quad \sigma_{2}=\left(\begin{array}{cc}
0 & -\mathrm{i} \\
\mathrm{i} & 0
\end{array}\right), \quad \sigma_{3}=\left(\begin{array}{cc}
1 & 0 \\
0 & -1
\end{array}\right) .
$$

The symbol $\mathbb{I}_{2}$ denotes the $2 \times 2$ identity matrix. The free-particle Dirac Hamiltonian with $\boldsymbol{A}(\boldsymbol{r}, t)=0$ and $\phi(\boldsymbol{r}, t)=0$ will be denoted by $\hat{H}_{0}$. The free-particle Dirac Hamiltonian features positive as well as negative energy-eigenvalues. We will also use the operator $\hat{p}_{0}$ to denote

$$
\hat{p}_{0}=\sqrt{\hat{H}_{0}^{2} / c^{2}}=\sqrt{m_{0}^{2} c^{2}+\hat{\boldsymbol{p}}^{2}},
$$

which has the same eigenstates as $\hat{H}_{0}$ but all its eigenvalues being positive. Furthermore, the matrices

$$
\Sigma_{i}=\left(\begin{array}{cc}
\sigma_{i} & 0 \\
0 & \sigma_{i}
\end{array}\right) \quad \text { for } i=1,2,3
$$

will be employed, which will be commonly combined into the three-component operator $\Sigma=\left(\Sigma_{1}, \Sigma_{2}, \Sigma_{3}\right)^{\top}$.

A relativistic spin operator may be introduced by splitting the undisputed total angular momentum operator $\hat{\boldsymbol{J}}$, which is in the Dirac representation

$$
\hat{\boldsymbol{J}}=\boldsymbol{r} \times(-\mathrm{i} \boldsymbol{\nabla})+\frac{1}{2} \hat{\boldsymbol{\Sigma}},
$$

into an external part $\hat{\boldsymbol{L}}$ and an internal part $\hat{\boldsymbol{S}}$, viz., $\hat{\boldsymbol{J}}=\hat{\boldsymbol{L}}+\hat{\boldsymbol{S}}$. These parts are commonly referred to as the orbital angular momentum and the spin. Because the orbital angular momentum operator $\hat{\boldsymbol{L}}$ is related to the position operator $\hat{\boldsymbol{r}}$ and the momentum operator $\hat{\boldsymbol{p}}=-\mathrm{i} \boldsymbol{\nabla}$ via $\hat{\boldsymbol{L}}=\hat{\boldsymbol{r}} \times \hat{\boldsymbol{p}}$, different definitions of the spin operator $\hat{\boldsymbol{S}}$ imply different relativistic position operators $\hat{\boldsymbol{r}}$. Thus, the question for the right splitting of the total angular momentum into an orbital part and a spin part is closely related to the quest for the right relativistic position operator [21-23].

There are two complementary approaches to determine a suitable relativistic spin operator from a set of possible candidates. A common approach is to judge a spin operator candidate by its mathematical properties. This means to analyze its symmetries, its behavior under various transformations, its algebraic features, and so on. Historically, most relativistic spin operators have been proposed on purely mathematical arguments. Considering that relativistic spin effects are expected to be detectable at highintensity laser facilities one may also compare theoretical predictions based on various relativistic spin operators to experimental measurements. In this way, candidates for relativistic spin operators that are not compatible with measurements can be ruled out on the basis of experimental results, rather than solely by mathematical reasoning. Here, we will adopt both approaches.

Mathematically, we demand from a proper relativistic spin operator $\hat{S}=\left(\hat{S}_{1}, \hat{S}_{2}, \hat{S}_{3}\right)^{\top}$ and its three components the following features:

1. Each component of a spin operator should be a Hermitian operator.

2. The physical quantity that is represented by the operator $\hat{S}$ should not depend on the orientation of the chosen coordinate system. Thus, a spin operator must transform under rotations like a vector, which is ensured by fulfilling [50]

$$
\left[\hat{J}_{i}, \hat{S}_{j}\right]=\mathrm{i} \varepsilon_{i, j, k} \hat{S}_{k}
$$

with $\varepsilon_{i, j, k}$ denoting the Levi-Civita symbol. 


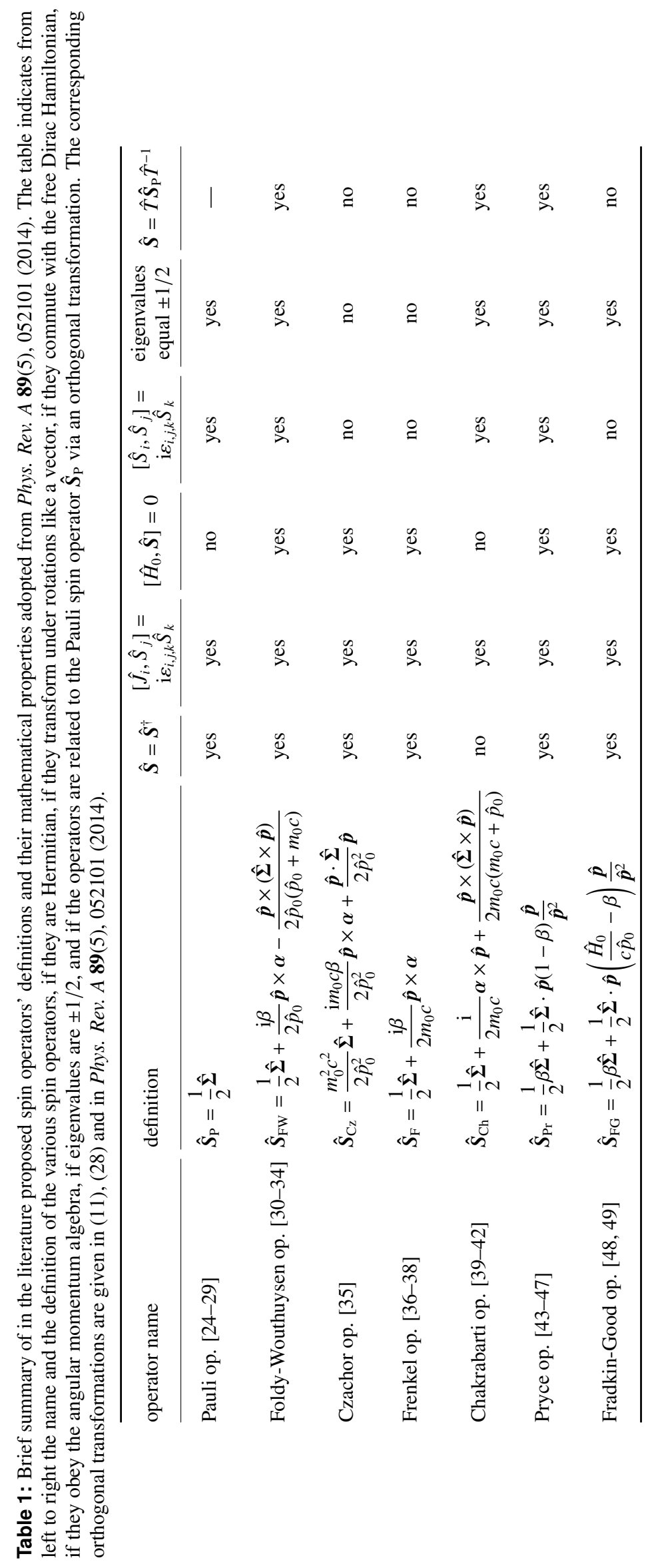


3. It is also required to commute with the free Dirac Hamiltonian, i. e., $\left[\hat{H}_{0}, \hat{S}\right]=0$. This property ensures that the relativistic spin operator is a constant of motion if forces are absent, such that spurious Zitterbewegung of the spin is prevented.

4. A spin operator must feature the two eigenvalues $\pm 1 / 2$ and it has to obey the angular momentum algebra

$$
\left[\hat{S}_{i}, \hat{S}_{j}\right]=\mathrm{i} \varepsilon_{i, j, k} \hat{S}_{k} .
$$

These two requirements are commonly regarded as the fundamental properties of angular momentum operators of spin one-half particles [50].

Table 1 gives an overview over several spin operators, which have been proposed in the literature, and presents their mathematical key features. A detailed description of these operators and their relations to each other is given elsewhere [18]. Note that some of these spin operators have been discovered by different authors in different contexts yielding different but mathematically equivalent forms. For example, the so-called Newton-Wigner spin operator [21], which may be written as [51]

$$
\hat{\boldsymbol{S}}_{\mathrm{FW}}=\frac{\hat{p}_{0}}{2 m_{0} c} \hat{\boldsymbol{\Sigma}}-\frac{\hat{\boldsymbol{p}} \cdot \hat{\boldsymbol{\Sigma}}}{2 m_{0} c\left(m_{0} c+\hat{p}_{0}\right)} \hat{\boldsymbol{p}}-\hat{\boldsymbol{p}} \times \boldsymbol{\alpha} \frac{\mathrm{i} \hat{H}_{0}}{2 m_{0} c^{2} \hat{p}_{0}},
$$

is just another way to express the Foldy-Wouthuysen spin operator given in Tab. 1

The Foldy-Wouthuysen, the Chakrabarti, the Pryce, and the Fradkin-Good spin operators are equivalent in the positive-energy subspace of free-particle states. This can be shown easily in the Foldy-Wouthuysen representation. The transition from the standard representation, where the Dirac Hamiltonian has the form (1) and the various spin operators have the forms given in Tab. 1. is mediated via the nonlocal unitary transform

$$
\hat{T}_{\mathrm{FW}}=\frac{\hat{p}_{0}+m_{0} c-\beta \boldsymbol{\alpha} \cdot \hat{\boldsymbol{p}}}{\sqrt{2 \hat{p}_{0}\left(\hat{p}_{0}+m_{0} c\right)}} .
$$

In the Foldy-Wouthuysen representation the free-particle Dirac Hamiltonian becomes diagonal,

$$
\hat{H}_{0}^{\prime}=\hat{T}_{\mathrm{FW}}^{-1} \hat{H}_{0} \hat{T}_{\mathrm{FW}}=c \beta \hat{p}_{0}
$$

and the Foldy-Wouthuysen spin operator has the form

$$
\hat{S}_{\mathrm{FW}}^{\prime}=\hat{T}_{\mathrm{FW}}^{-1} \hat{S}_{\mathrm{FW}} \hat{T}_{\mathrm{FW}}=\left(\begin{array}{cc}
\frac{1}{2} \sigma & 0 \\
0 & \frac{1}{2} \sigma
\end{array}\right) .
$$

Since the momentum operator is invariant under the Foldy-Wouthuysen transformation $\hat{T}_{\mathrm{FW}}$, the simultaneous eigenstates of the free-particle Dirac Hamiltonian, the momentum operator, and the $z$ component of the Foldy-Wouthuysen spin operator are in the Foldy-Wouthuysen representation given by

$$
\boldsymbol{s}_{\mathrm{FW},+, \boldsymbol{p}, \uparrow}=\left(\begin{array}{l}
1 \\
0 \\
0 \\
0
\end{array}\right) \mathrm{e}^{\mathrm{i} \boldsymbol{p} \cdot \boldsymbol{r},} \quad \boldsymbol{s}_{\mathrm{FW},+, \boldsymbol{p} \downarrow \downarrow}=\left(\begin{array}{l}
0 \\
1 \\
0 \\
0
\end{array}\right) \mathrm{e}^{\mathrm{i} \boldsymbol{p} \cdot \boldsymbol{r}}, \quad \boldsymbol{s}_{\mathrm{FW},-, \boldsymbol{p}, \uparrow}=\left(\begin{array}{l}
0 \\
0 \\
1 \\
0
\end{array}\right) \mathrm{e}^{\mathrm{i} \boldsymbol{p} \cdot \boldsymbol{r}}, \quad \boldsymbol{s}_{\mathrm{FW},-, \boldsymbol{p}, \downarrow}=\left(\begin{array}{l}
0 \\
0 \\
0 \\
1
\end{array}\right) \mathrm{e}^{\mathrm{i} \boldsymbol{p} \cdot \boldsymbol{r}},
$$

where the indices indicate the sign of the energy eigenvalue, the momentum eigenvalues and the spin eigenvalue $(\uparrow$ for $1 / 2$, $\downarrow$ for $-1 / 2$ ). In the Foldy-Wouthuysen representation the Chakrabarti, the Pryce, and the Fradkin-Good spin operators are given by the rather simple expressions

$$
\begin{aligned}
& \hat{\boldsymbol{S}}_{\mathrm{Ch}}^{\prime}=\hat{T}_{\mathrm{FW}}^{-1} \hat{\boldsymbol{S}}_{\mathrm{Ch}} \hat{T}_{\mathrm{FW}}=\left(\begin{array}{cc}
\frac{1}{2} \sigma & \mathrm{i} \frac{\sigma \times \hat{p}}{m_{0} c} \\
0 & \frac{1}{2} \sigma
\end{array}\right), \\
& \hat{\boldsymbol{S}}_{\mathrm{Pr}}^{\prime}=\hat{T}_{\mathrm{FW}}^{-1} \hat{\boldsymbol{S}}_{\mathrm{Pr}} \hat{T}_{\mathrm{FW}}=\left(\begin{array}{cc}
\frac{1}{2} \boldsymbol{\sigma} & 0 \\
0 & -\frac{1}{2} \sigma+\frac{\sigma \cdot \hat{p}}{\hat{p}^{2}} \hat{\boldsymbol{p}}
\end{array}\right) \text {, } \\
& \hat{\boldsymbol{S}}_{\mathrm{FG}}^{\prime}=\hat{T}_{\mathrm{FW}}^{-1} \hat{\boldsymbol{S}}_{\mathrm{FG}} \hat{T}_{\mathrm{FW}}=\left(\begin{array}{cc}
\frac{1}{2} \sigma & 0 \\
0 & -\frac{1}{2} \sigma
\end{array}\right) \text {. }
\end{aligned}
$$

Because the operators (13), (15), (16), and (17) have the same upper left $2 \times 2$ matrix, all these operators act in the same way on the positive-energy free particle states given in (14). For quantum states that are superpositions of free-particle states with positive and negative energy, however, these spin operators are not equivalent. 
On the basis of the four criteria given above, one may argue that only the Foldy-Wouthuysen spin operator and the Pryce spin operator qualify as proper relativistic spin operators because only these two fulfill all four criteria. However, the question of which of the proposed relativistic spin operators in Tab. 1 provides the correct mathematical description of spin can be answered definitely only by comparing theoretical predictions with experimental results. Because if an interaction with some external fields is introduced a superposition of positive-energy free-particle states evolves such that negative-energy free-particle states become populated. The proposed spin operators are not equivalent if they are applied to positive-energy states of Hamiltonians with nonvanishing electromagnetic fields. Therefore, it becomes possible to distinguish between the various spin operators by determining their expectation values for electrons interacting with electromagnetic fields.

For this purpose it is desirable to employ a physical system that shows strong relativistic effects and is as simple as possible. Such a setup is provided by the bound eigenstates of highly charged hydrogen-like ions, i. e., atomic systems with an atomic core of $Z$ protons and a single electronic charge. These ions can be produced at storage rings [52] or by utilizing electron beam ion traps [53, 54] up to $Z=92$ (hydrogen-like uranium). The degenerate bound eigenstates of the corresponding Coulomb-Dirac Hamiltonian (in atomic units)

$$
\hat{H}_{C}=\hat{H}_{0}-\frac{Z}{|\boldsymbol{r}|}
$$

are commonly expressed as simultaneous eigenstates $\psi_{n, j, m, \kappa}$ of $\hat{H}_{C}, \hat{\boldsymbol{J}}^{2}, \hat{J}_{3}$, and the so-called spin-orbit operator $\hat{K}=$ $\beta\{\hat{\boldsymbol{\Sigma}} \cdot[\boldsymbol{r} \times(-\mathrm{i} \boldsymbol{\nabla})+1)]\}$ fulfilling the eigenequations [55, 56]

$$
\begin{aligned}
\hat{H}_{C} \psi_{n, \kappa, j, m} & =\mathcal{E}(n, \kappa) \psi_{n, \kappa, j, m}, & n & =1,2, \ldots, \\
\hat{K} \psi_{n, \kappa, j, m} & =\kappa \psi_{n, \kappa, j, m}, & |\kappa| & =1,2, \ldots, n, \kappa \neq-n, \\
\hat{\boldsymbol{J}}^{2} \psi_{n, \kappa, j, m} & =j(j+1) \psi_{n, \kappa, j, m}, & j & =|\kappa|-\frac{1}{2}, \\
\hat{J}_{3} \psi_{n, \kappa, j, m} & =m \psi_{n, \kappa, j, m}, & m & =-j,(j-1), \ldots, j .
\end{aligned}
$$

The eigenenergies are given with $\alpha_{\mathrm{el}}$ denoting the fine structure constant by

$$
\mathcal{E}(n, j)=m_{0} c^{2}\left[1+\left(\frac{\alpha_{\mathrm{el}}^{2} Z^{2}}{n-j-1 / 2+\sqrt{(j-1 / 2)^{2}-\alpha_{\mathrm{el}}^{2} Z^{2}}}\right)\right]^{-1 / 2} .
$$

The degenerate hydrogenic ground state is with $\gamma=\sqrt{1-Z^{2} \alpha_{\mathrm{el}}^{2}}$, the radial function

$$
\psi(r)=\frac{\mathrm{e}^{-m_{0} Z r}}{\left(2 m_{0} Z r\right)^{1-\gamma}},
$$

and the normalizing factor

$$
\mathcal{N}=\left(2 m_{0} Z\right)^{3 / 2} \sqrt{\frac{1+\gamma}{2 \Gamma(1+2 \gamma)}}
$$

given by the two wave functions [57]

$$
\begin{aligned}
& \psi_{1,1, \frac{1}{2}, \frac{1}{2}}(r, \theta, \phi)=\mathcal{N} \psi(r)\left(\begin{array}{c}
Y_{0,0}(\theta, \phi) \\
0 \\
\mathrm{i} \frac{1-\gamma}{Z \alpha_{\mathrm{el}}} \sqrt{\frac{1}{3}} Y_{1,0}(\theta, \phi) \\
-\mathrm{i} \frac{1-\gamma}{Z \alpha_{\mathrm{el}}} \sqrt{\frac{2}{3}} Y_{1,1}(\theta, \phi)
\end{array}\right), \\
& 0 \\
& Y_{0,0}(\theta, \phi) \\
& \psi_{1,1, \frac{1}{2},-\frac{1}{2}}(r, \theta, \phi)=\mathcal{N} \psi(r)\left(\begin{array}{c}
1-\gamma \\
\mathrm{i} \frac{2}{\mathrm{Z}} Y_{\mathrm{el}} \\
-\mathrm{i} \frac{1-\gamma}{Z \alpha_{\mathrm{el}}} \sqrt{\frac{1}{3}} Y_{1,0}(\theta, \phi)
\end{array}\right) .
\end{aligned}
$$

The spin expectation values of the $z$ component of the in Tab. 1 defined spin operators are presented in Fig. 1 a). For small atomic numbers $(Z \lesssim 20$ ), all spin operators yield about $1 / 2$; for larger $Z$ when relativistic effects set in, however, expectation values differ significantly from each other. While for Pauli, Fouldy-Wouthuysen, Czachor, Chakrabarti, and Fradkin-Good spin 

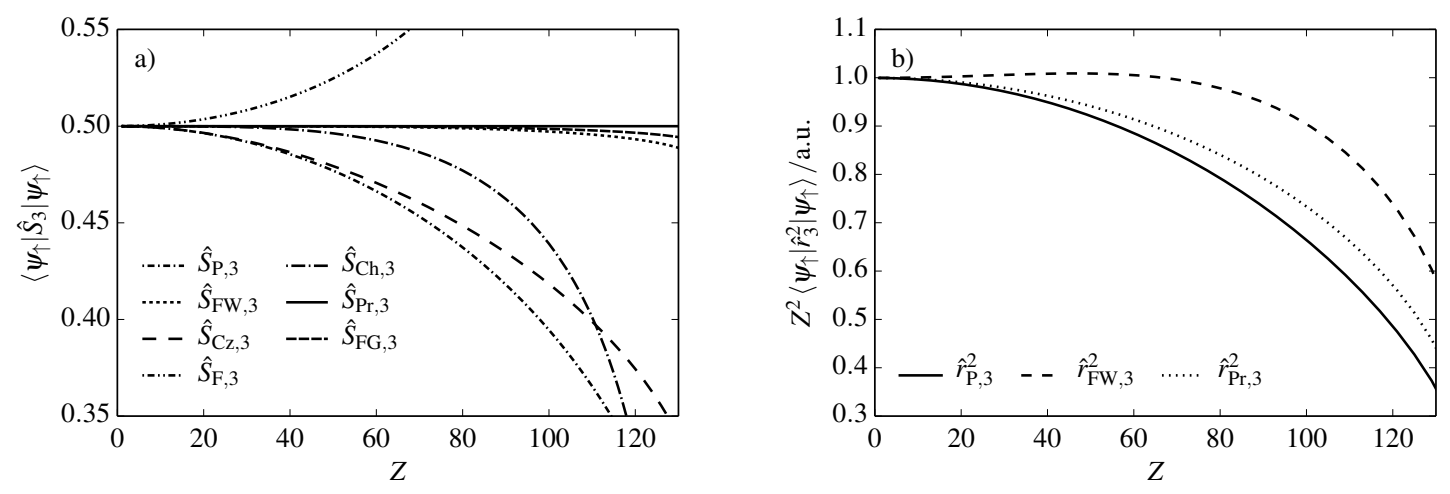

Figure 1: (a) Spin expectation values of various relativistic spin operators for the hydrogenic ground state 23a as a function of the atomic number Z, adopted from New J. Phys. 16(4), 043012 (2014). For the ground state 23b) we find the same spin expectation values but with opposite sign (not displayed in the plot). (b) Expectation values of the position variance with respect to the $z$ coordinate for the hydrogenic ground state 23a as a function of the atomic number $Z$. The wave function's variance depends on the definition of the relativistic position operator, which is induced by the definition of the relativistic spin operator.

operators the spin expectation value is reduced, the expectation value of the Frenkel spin operator exceeds $1 / 2$. Only for the Pryce operator we find that the spin expectation values is $1 / 2$ for all values of $Z$, which can be also shown analytically [18]. A comparison of the results of a spin-measurement experiment for groundstate electrons in hydrogen-like highly-charged ions to the numerical results Fig. 1] a) would allow to find a suitable relativistic spin operator or at least to rule out some candidates [17].

As outlined above, each definition of a relativistic spin operator $\hat{\boldsymbol{S}}$ induces also a relativistic position operator $\hat{\boldsymbol{r}}$. Because only the Fouldy-Wouthuysen and the Pryce operators fulfill all our mathematical criteria for a proper spin operator we focus on these two in the following. For determining the corresponding position operator the defining relation

$$
\hat{\boldsymbol{J}}=\boldsymbol{r} \times(-\mathrm{i} \boldsymbol{\nabla})+\boldsymbol{\Sigma} / 2=\hat{\boldsymbol{r}} \times(-\mathrm{i} \boldsymbol{\nabla})+\hat{\boldsymbol{S}}
$$

is rather unwieldy. In the case of the Fouldy-Wouthuysen and the Pryce operators it is more convenient to utilize the fact that these are related to the Pauli spin operator via a unitary transform. The position operator of the Pauli spin operator is just

$$
\hat{\boldsymbol{r}}_{\mathrm{P}}=\boldsymbol{r} .
$$

Therefore, the position operators of the Fouldy-Wouthuysen and the Pryce operators are given by

$$
\hat{\boldsymbol{r}}_{\mathrm{FW}}=\hat{T}_{\mathrm{FW}} \boldsymbol{r} \hat{T}_{\mathrm{FW}}^{-1}
$$

and

$$
\hat{\boldsymbol{r}}_{\mathrm{Pr}}=\hat{T}_{\mathrm{Pr}} \boldsymbol{r} \hat{T}_{\mathrm{Pr}}^{-1}
$$

with the transformations $\hat{T}_{\mathrm{FW}}$ and $\hat{T}_{\mathrm{Pr}}$ defined in (11) and by

$$
\hat{T}_{\mathrm{Pr}}=\left(\begin{array}{cc}
\mathbb{I}_{2} & 0 \\
0 & \mathrm{i} \frac{\boldsymbol{\sigma} \cdot \hat{\boldsymbol{p}}}{|\hat{\boldsymbol{p}}|}
\end{array}\right) .
$$

Determining an explicit expression for the transformation (26) yields the famous Fouldy-Wouthuysen mean position operator [30][58]

$$
\hat{\boldsymbol{r}}_{\mathrm{FW}}=\boldsymbol{r}+\mathrm{i} \hbar\left(\frac{\mathrm{i} \hat{\boldsymbol{\Sigma}} \times \hat{\boldsymbol{p}}}{2 \hat{p}_{0}\left(\hat{p}_{0}+m_{0} c\right)}-\frac{\beta(\boldsymbol{\alpha} \cdot \hat{\boldsymbol{p}}) \hat{\boldsymbol{p}}}{2 \hat{p}_{0}^{2}\left(\hat{p}_{0}+m_{0} c\right)}+\frac{\beta \boldsymbol{\alpha}}{2 \hat{p}_{0}}\right),
$$

which is equivalent to the Newton-Wigner position operator [21], while [27] yields

$$
\hat{\boldsymbol{r}}_{\mathrm{Pr}}=\boldsymbol{r}-\hbar\left(\begin{array}{cc}
0 & 0 \\
0 & \frac{\boldsymbol{\sigma} \times \hat{\boldsymbol{p}}}{|\hat{\boldsymbol{p}}|^{2}}
\end{array}\right) .
$$


For numerical calculations of expectation values, however, it is more convenient to utilize the forms (26) and (27).

Because of symmetry reasons the expectation values of the position operators $\hat{\boldsymbol{r}}_{\mathrm{P}}, \hat{\boldsymbol{r}}_{\mathrm{FW}}$, and $\hat{\boldsymbol{r}}_{\mathrm{Pr}}$ are zero if these position operators are applied to the hydrogenic ground states (23a) and (23b). The second moment, however, does not vanish. The variance of a nonrelativistic hydrogenic ground state scales with $1 / Z^{2}$. Due to relativistic effects the ground states' wavepackets shrink even faster. How fast, depends on the definition of the position operator, as shown Fig. 1 b). In principle, one can measure the variance of the ground state of a highly charged ion and compare it to the predictions in Fig. 1 b) and in this way determine the correct relativistic position operator and indirectly also the correct relativistic spin operator.

\section{Electron-spin precession in elliptically polarized light}

In the previous section we argued that the spin of free electrons should be modeled by the Fouldy-Wouthuysen spin operator. In the following we will utilize this operator to study a relativistic spin dynamics, which originates from a coupling of the electron's spin to the spin of an electromagnetic wave with elliptical polarization.

The electric and magnetic field components of two elliptically polarized laser fields propagating into the positive or negative direction of the $x$ axis are given by

$$
\begin{aligned}
& \boldsymbol{E}_{1,2}(\boldsymbol{r}, t)=\hat{E}\left(\cos \frac{2 \pi(x \mp c t)}{\lambda} \boldsymbol{e}_{y}+\cos \left(\frac{2 \pi(x \mp c t)}{\lambda} \pm \eta\right) \boldsymbol{e}_{z}\right) \\
& \boldsymbol{B}_{1,2}(\boldsymbol{r}, t)=\frac{\hat{E}}{c}\left(\mp \cos \left(\frac{2 \pi(x \mp c t)}{\lambda} \pm \eta\right) \boldsymbol{e}_{y} \pm \cos \frac{2 \pi(x \mp c t)}{\lambda} \boldsymbol{e}_{z}\right) .
\end{aligned}
$$

Here, the position vector $\boldsymbol{r}=(x, y, z)^{\top}$, the time $t$, and $\boldsymbol{e}_{x}, \boldsymbol{e}_{y}$, and $\boldsymbol{e}_{z}$ denoting unit vectors in the direction of the coordinate axes are used. The parameter $\eta \in(-\pi, \pi]$ determines the degree of the light beams' ellipticity with $\eta=0$ and $\eta=\pi$ corresponding to linear polarization and $\eta= \pm \pi / 2$ to circular polarization. The two electromagnetic waves (31) feature the same wavelength $\lambda$, the same electric field amplitude $\hat{E}$, and the same intensity

$$
I=\varepsilon_{0} c \hat{E}^{2}
$$

but have opposite helicity. Introducing the wave number $k=2 \pi / \lambda$ and the lasers fields' angular frequency $\omega=k c$, the Coulomb gauge vector potentials $\boldsymbol{A}_{1,2}(\boldsymbol{r}, t)$ of the elliptically polarized fields 31 ) are

$$
\boldsymbol{A}_{1,2}(\boldsymbol{r}, t)=-\frac{\hat{E}}{\omega}\left(\mp \sin (k x \mp \omega t) \boldsymbol{e}_{y} \mp \sin (k x \mp \omega t \pm \eta) \boldsymbol{e}_{z}\right) .
$$

Each of the electromagnetic fields specified by (31) carries the photonic spin density

$$
\varepsilon_{0} \boldsymbol{E}_{1,2} \times \boldsymbol{A}_{1,2}=\frac{\varepsilon_{0} \hat{E}^{2} \lambda \sin \eta}{2 \pi c} \boldsymbol{e}_{x} .
$$

As one can show via the Volkov solution of the Dirac equation[59] a single plane wave as given in (31) cannot change the spin orientation of an electron. Therefore, we consider a standing wave, which is formed by superimposing the two counterpropagating waves given in 31. The magnetic vector potential of the combined laser fields is given by

$$
\boldsymbol{A}(\boldsymbol{r}, t)=-\frac{2 w(t) \hat{E}}{\omega} \cos k x\left(\sin \omega t \boldsymbol{e}_{y}+\sin (\omega t-\eta) \boldsymbol{e}_{z}\right)
$$

Here the window function

$$
w(t)= \begin{cases}\sin ^{2} \frac{\pi t}{2 \Delta T} & \text { if } 0 \leq t \leq \Delta T \\ 1 & \text { if } \Delta T \leq t \leq T-\Delta T \\ \sin ^{2} \frac{\pi(T-t)}{2 \Delta T} & \text { if } T-\Delta T \leq t \leq T\end{cases}
$$

was introduced to allow for a smooth turn-on and turn-off of the laser field. The parameters $T$ and $\Delta T$ denote the total interaction time and the turn-on and turn-off intervals. For circularly polarized plane waves $(\eta=\pi / 2)$, the electric and the magnetic components of the standing wave are parallel to each other and rotate around the propagation direction. The maxima of the electric and the magnetic field components are shifted against eachother by $\lambda / 4$.

Solving the time-dependent Dirac equation till time $t=T$ with a common eigenstate of the free Dirac Hamiltonian, the momentum operator, and the $z$-component of the Foldy-Wouthuysen spin operator with zero momentum and positive spin as 


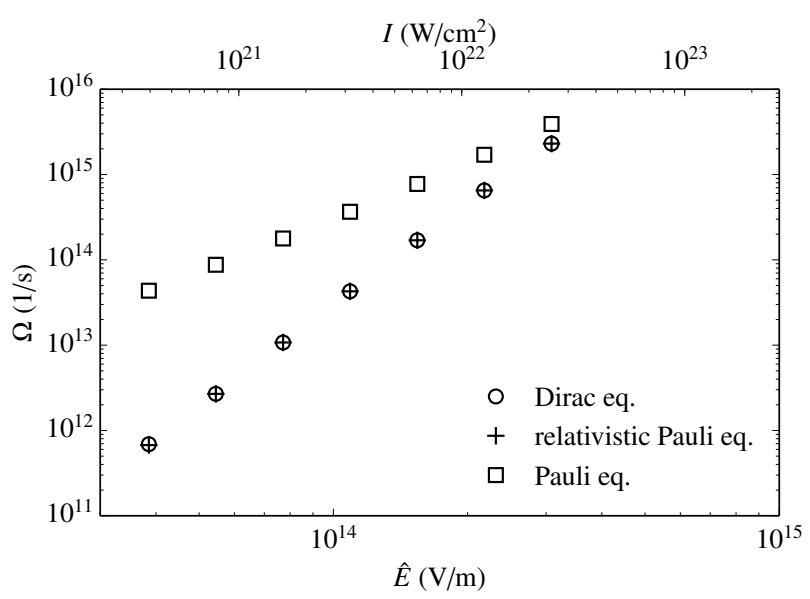

Figure 2: Angular frequency $\Omega$ of the spin precession as a function of the laser's electric field strength $\hat{E}$ and its intensity $I$ for electromagnetic fields with a wavelength $\lambda=0.159 \mathrm{~nm}$. Depending on the applied theory (the Dirac equation (1), the relativistic Pauli equation 38, or the nonrelativistic Pauli equation) the spin of an electron in two counterpropagating circularly polarized light waves scales with the second (Pauli equation) or the fourth power (Dirac equation, relativistic Pauli equation) of $\hat{E}$. Numerical data adopted from New J. Phys. 16(4), 043012 (2014).

initial condition shows that the electron's spin precesses around the propagation axis of the electromagnetic fields [19, 20]. The role of the photonic spin density for the electronic spin precession becomes evident by considering the weakly relativistic limit of the Dirac equation (1]. In this limit, this equation reduces via a Foldy-Wouthuysen transformation [30, 31, 60] to

$$
\begin{aligned}
\mathrm{i} \hbar \dot{\Psi}(\boldsymbol{r}, t) & =\left(\frac{(-\mathrm{i} \hbar \boldsymbol{\nabla}-q \boldsymbol{A}(\boldsymbol{r}, t))^{2}}{2 m_{0}}-\frac{q \hbar}{2 m_{0}} \boldsymbol{\sigma} \cdot \boldsymbol{B}(\boldsymbol{r}, t)+q \phi(\boldsymbol{r}, t)-\frac{(-\mathrm{i} \hbar \boldsymbol{\nabla}-q \boldsymbol{A}(\boldsymbol{r}, t))^{4}}{8 m_{0}^{3} c^{2}}-\frac{q^{2} \hbar^{2}}{8 m_{0}^{3} c^{4}}\left(c^{2} \boldsymbol{B}(\boldsymbol{r}, t)^{2}-\boldsymbol{E}(\boldsymbol{r}, t)^{2}\right)\right. \\
& \left.-\frac{q \hbar}{4 m_{0}^{2} c^{2}} \boldsymbol{\sigma} \cdot(\boldsymbol{E}(\boldsymbol{r}, t) \times(-\mathrm{i} \hbar \boldsymbol{\nabla}-q \boldsymbol{A}(\boldsymbol{r}, t)))-\frac{q \hbar^{2}}{8 m_{0}^{2} c^{2}} \boldsymbol{\nabla} \cdot \boldsymbol{E}(\boldsymbol{r}, t)+\frac{q \hbar}{8 m_{0}^{3} c^{2}}\left\{\boldsymbol{\sigma} \cdot \boldsymbol{B}(\boldsymbol{r}, t),(-\mathrm{i} \hbar \boldsymbol{\nabla}-q \boldsymbol{A}(\boldsymbol{r}, t))^{2}\right\}\right) \Psi(\boldsymbol{r}, t)
\end{aligned}
$$

for the now two-component wave function $\Psi(\boldsymbol{r}, t)$ with the vector potential $\boldsymbol{A}(\boldsymbol{r}, t)$ given by (35) and the electromagnetic fields $\boldsymbol{B}(\boldsymbol{r}, t)=\boldsymbol{\nabla} \times \boldsymbol{A}(\boldsymbol{r}, t)$ and $\boldsymbol{E}(\boldsymbol{r}, t)=-\dot{\boldsymbol{A}}(\boldsymbol{r}, t)$. In leading order Eq. (37) features four terms that may cause spin dynamics. The so-called Zeeman term $\sim \boldsymbol{\sigma} \cdot \boldsymbol{B}(\boldsymbol{r}, t)$ and its the lowest-order relativistic correction given by the anticommutator expression $\sim\left\{\boldsymbol{\sigma} \cdot \boldsymbol{B}(\boldsymbol{r}, t),(-\mathrm{i} \hbar \boldsymbol{\nabla}-q \boldsymbol{A}(\boldsymbol{r}, t))^{2}\right\}$ mediate the coupling of the electron's spin to the magnetic field. The term $\sim \boldsymbol{\sigma} \cdot \boldsymbol{E}(\boldsymbol{r}, t) \times \mathrm{i} \hbar \boldsymbol{\nabla}$ leads to the so-called spin-orbit interaction, i. e., the coupling between the electron's spin and its orbital angular momentum. The term $\sim \boldsymbol{\sigma} \cdot(\boldsymbol{E}(\boldsymbol{r}, t) \times \boldsymbol{A}(\boldsymbol{r}, t))$ may be interpreted as a coupling of the spin density of the external electromagnetic wave to the electron's spin. Considering the relativistic correction due to the electromagnetic wave's spin density as the only relativistic correction to the nonrelativistic Pauli equation the relativistic electron motion in the vector potential (35) may be described by the relativistic Pauli equation [19, 20]

$$
\mathrm{i} \hbar \dot{\Psi}(\boldsymbol{r}, t)=\left(\frac{1}{2 m_{0}}(-\mathrm{i} \hbar \boldsymbol{\nabla}-q \boldsymbol{A}(\boldsymbol{r}, t))^{2}-\frac{q \hbar}{2 m_{0}} \boldsymbol{\sigma} \cdot \boldsymbol{B}(\boldsymbol{r}, t)+\frac{q^{2} \hbar}{4 m_{0}^{2} c^{2}} \boldsymbol{\sigma} \cdot(\boldsymbol{E}(\boldsymbol{r}, t) \times \boldsymbol{A}(\boldsymbol{r}, t))\right) \Psi(\boldsymbol{r}, t) .
$$

Numerical calculations indicate that the relativistic Pauli equation (38) is sufficient to reproduce the spin dynamics of the fully relativistic Dirac equation (1). The nonrelativistic Pauli equation, however, yields a completely different spin dynamics as shown in Fig. 2. Depending on if the Dirac equation (1), the relativistic Pauli equation (38), or the nonrelativistic Pauli equation is applied, the spin of an electron in two counterpropagating circularly polarized light waves scales with the second (Pauli equation) or the fourth power (Dirac equation, relativistic Pauli equation) of the electric field's amplitude $\hat{E}$. Further analytical calculations based on the Dirac equation and time-dependent perturbation theory [20] show that the electron's spin precesses with an angular frequency that is proportional to the photonic spin density $\varrho_{\sigma}=\varepsilon_{0} \hat{E}^{2} \lambda /(\pi c)$, the laser field's intensity $I$ given in [32], and the fourth power of the wavelength:

$$
\Omega=\varrho_{\sigma} I \lambda^{4} \frac{\alpha_{\mathrm{el}}^{2}}{2 \pi^{2} m_{0}^{2} c^{3}}
$$

The proportionality factor $\alpha_{\mathrm{el}}^{2} /\left(2 \pi^{2} m_{0}^{2} c^{3}\right)$ is independent of the standing wave's electromagnetic field. 
a)

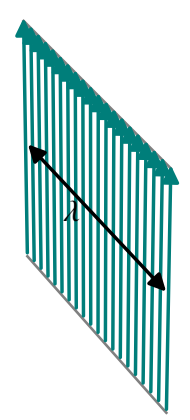

b)

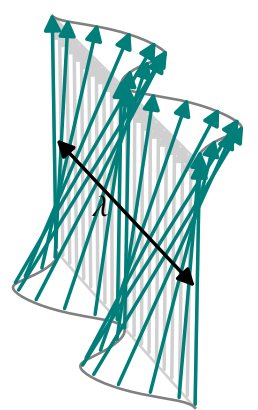

c)

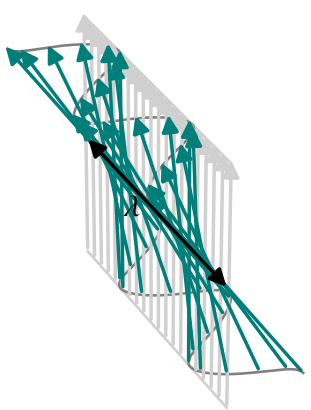

Figure 3: An ensemble of classical spins under the effect of a magnetic field and a photonic spin density of a standing wave formed by two counterpropagating circularly polarized waves. Part a): Initially spins are aligned. Part b): The rotating magnetic field causes a positiondependent rotation of the spin vectors. Part c): Also the photonic spin density causes a spin rotation but into the opposite direction. Averaged over a wavelength both effects cancel eachother.

The role of the relativistic correction due to the photonic spin density is pivotal, its influence on the scaling of the spin precession frequency does not become small in the limit of weak fields, which is usually related to a nonrelativistic limit. The nonexistence of a nonrelativistic limit results because the Zeeman term is due to the fast oscillation of the magnetic field effectively as strong as the relativistic correction due to the photonic spin density. On the basis of a classical argument [19] one can show that the effects of the Zeeman term and the correction due to the photonic spin density on the motion of the electron spin counteract eachother. The initial electron's quantum state, which is a momentum eigenstate, is delocalized over several laser wavelengths. Thus, we mimic the quantum wavepacket by an ensemble of classical particles with spin angular momentum. These particles are placed along the $x$ axis with initially aligned spin direction, see also Fig. 3 3 a). The dynamics of a classical electron spin $\boldsymbol{s}$ at fixed position $\boldsymbol{r}$ in the magnetic field $\boldsymbol{B}(\boldsymbol{r}, t)=\boldsymbol{B}_{1}(\boldsymbol{r}, t)+\boldsymbol{B}_{2}(\boldsymbol{r}, t)$ is governed by the classical equation of motion

$$
\dot{\boldsymbol{s}}(t)=\frac{q}{m_{0}} \boldsymbol{s}(t) \times \boldsymbol{B}(\boldsymbol{r}, t)=\frac{2 q \hat{E} \cos k x}{m_{0}} \boldsymbol{s}(t) \times\left(\cos \omega t \boldsymbol{e}_{y}+\sin \omega t \boldsymbol{e}_{y}\right) .
$$

In a static and homogeneous magnetic field of strength $\hat{E} / c$, the electron's spin would precess around an axis parallel to the magnetic field's direction with the angular frequency $\Omega_{\mathrm{L}}=q \hat{E} /\left(m_{0} c\right)$. For a rotating magnetic field and parameters such that $\Omega_{\mathrm{L}} \ll \omega$, however, the magnetic field rotates so fast that the spin precesses around the rotation axis of the magnetic field. For the magnetic field of a standing wave formed by two counterpropagating circularly polarized waves given by the vector potential (35) a position-dependent angular frequency $2 \Omega_{\mathrm{P}} \sin ^{2} k x$ results with $\Omega_{\mathrm{P}}=(q \hat{E})^{2} \lambda /\left(2 \pi m_{0}^{2} c^{3}\right)$, see Fig. $\left.3 \mathrm{~b}\right)$. Similarly, the relativistic correction due to the photonic spin density in 38 to the Pauli equation leads to the classical equation

$$
\dot{\boldsymbol{s}}(t)=-\frac{q^{2}}{2 m_{0}^{2} c^{2}} \boldsymbol{s}(t) \times(\boldsymbol{E}(\boldsymbol{r}, t) \times \boldsymbol{A}(\boldsymbol{r}, t))=-\frac{(q \hat{E})^{2} \lambda \cos ^{2} k x}{\pi m_{0}^{2} c^{3}} \boldsymbol{s}(t) \times \boldsymbol{e}_{x} .
$$

This yields also a position-dependent angular frequency, but now $-2 \Omega_{\mathrm{P}} \cos ^{2} k x$, see Fig. 3 c). As a consequence, a classical spin under the effect of both spin terms, the Zeeman term and the relativistic term due to the photonic spin density, rotates in a short time interval $\Delta t$ around an angle of about $2 \Omega_{\mathrm{P}}\left(\sin ^{2} k x-\cos ^{2} k x\right) \Delta t$. Averaged over a laser wavelength this rotation angle vanishes and the effects of the two spin terms chancel each other in our classical model. Thus, the classical model explains how the effect of the laser fields' spin density leads to a breakdown of the quadratic scaling of the spin-precession angular frequency in $\hat{E}$ that results if only the magnetic field is taken into account. The model, however, is not able to reproduce the quartic scaling in $\hat{E}$ that results from the fully relativistic quantum mechanical Dirac equation. The quartic scaling results as a genuine quantum effect from the fast temporal oscillations combined with the spatial modulation of the electromagnetic fields. In fact, further numerical calculations show that the quantum mechanical wavepacket accumulates in regions of the standing light wave with high magnetic fields [20].

[1] M. Altarelli, R. Brinkmann, M. Chergui, W. Decking, B. Dobson, S. Düsterer, G. Grübel, W. Graeff, H. Graafsma, J. Hajdu, J. Marangos, J. Pflüger, H. Redlin, D. Riley, I. Robinson, J. Rossbach, A. Schwarz, K. Tiedtke, T. Tschentscher, I. Vartaniants, H. Wabnitz, H. Weise, 
R. Wichmann, K. Witte, A. Wolf, M. Wulff, and M. Yurkov, eds., The European X-Ray Free-Electron Laser Technical design report (DESY XFEL Project Group European XFEL Project Team Deutsches Elektronen-Synchrotron Member of the Helmholtz Association, Hamburg, 2007).

[2] V. Yanovsky, V. Chvykov, G. Kalinchenko, P. Rousseau, T. Planchon, T. Matsuoka, A. Maksimchuk, J. Nees, G. Cheriaux, G. Mourou, and K. Krushelnick, Opt. Express 16, 2109 (2008).

[3] B. W. J. McNeil and N. R. Thompson, Nat. Photonics 4, 814 (2010)

[4] P. Emma, R. Akre, J. Arthur, R. Bionta, C. Bostedt, J. Bozek, A. Brachmann, P. Bucksbaum, R. Coffee, F.-J. Decker, Y. Ding, D. Dowell, S. Edstrom, A. Fisher, J. Frisch, S. Gilevich, J. Hastings, G. Hays, P. Hering, Z. Huang, R. Iverson, H. Loos, M. Messerschmidt, A. Miahnahri, S. Moeller, H.-D. Nuhn, G. Pile, D. Ratner, J. Rzepiela, D. Schultz, T. Smith, P. Stefan, H. Tompkins, J. Turner, J. Welch, W. White, J. Wu, G. Yocky, and J. Galayda, Nat. Photonics 4, 641 (2010)

[5] G. Mourou, N. Fisch, V. Malkin, Z. Toroker, E. Khazanov, A. Sergeev, T. Tajima, and B. Le Garrec, Opt. Commun. 285, 720 (2012)

[6] F. Ehlotzky, K. Krajewska, and J. Z. Kamiński, Rep. Prog. Phys. 72, 046401 (2009)

[7] A. Di Piazza, C. Müller, K. Z. Hatsagortsyan, and C. H. Keitel, Rev. Mod. Phys. 84, 1177 (2012)

[8] D. Blaschke, A. Prozorkevich, C. Roberts, S. Schmidt, and S. Smolyansky, Phys. Rev. Lett. 96, 140402 (2006)

[9] A. Bell and J. Kirk, Phys. Rev. Lett. 101, 200403 (2008).

[10] O. J. Pike, F. Mackenroth, E. G. Hill, and S. J. Rose, Nat. Photonics 8, 434 (2014)

[11] M. W. Walser, D. J. Urbach, K. Z. Hatsagortsyan, S. X. Hu, and C. H. Keitel, Phys. Rev. A 65, 043410 (2002).

[12] F. Faisal and S. Bhattacharyya, Phys. Rev. Lett. 93, 053002 (2004)

[13] G. Brodin, M. Marklund, J. Zamanian, and M. Stefan, Plasma Phys. Controlled Fusion 53, 074013 (2011)

[14] S. Ahrens, H. Bauke, C. H. Keitel, and C. Müller, Phys. Rev. Lett. 109, 043601 (2012)

[15] S. Ahrens, T.-O. Müller, S. Villalba-Chávez, H. Bauke, and C. Müller, Journal of Physics: Conference Series 414, 012012 (2013)

[16] M. Klaiber, E. Yakaboylu, C. Müller, H. Bauke, G. G. Paulus, and K. Z. Hatsagortsyan, J. Phys. B: At., Mol. Opt. Phys. 47, 065603 (2014)

[17] H. Bauke, S. Ahrens, C. H. Keitel, and R. Grobe, New J. Phys. 16, 043012 (2014)

[18] H. Bauke, S. Ahrens, C. H. Keitel, and R. Grobe, Phys. Rev. A 89, 052101 (2014)

[19] H. Bauke, S. Ahrens, C. H. Keitel, and R. Grobe, New J. Phys. 16, 103028 (2014)

[20] H. Bauke, S. Ahrens, and R. Grobe, Phys. Rev. A 90, 052101 (2014)

[21] T. D. Newton and E. P. Wigner, Rev. Mod. Phys. 21, 400 (1949)

[22] T. F. Jordan and N. Mukunda, Phys. Rev. 132, 1842 (1963)

[23] R. O'Connell and E. Wigner, Phys. Lett. A 67, 319 (1978)

[24] E. L. Hill and R. Landshoff, Rev. Mod. Phys. 10, 87 (1938)

[25] P. A. M. Dirac, The Principles of Quantum Mechanics, 4th ed., International Series of Monographs on Physics, Vol. 27 (Oxford University Press, Oxford, 1958).

[26] P. A. M. Dirac, Proc. R. Soc. London, Ser. A 322, 435 (1971)

[27] H. C. Ohanian, Amer. J. Phys. 54, 500 (1986)

[28] E. M. Lifshitz, L. P. Pitaevskii, and V. B. Berestetskii, Quantum Electrodynamics, 2nd ed., Course of Theoretical Physics, Vol. 4 (Butterworth Heinemann, Oxford, 1996).

[29] R. P. Feynman, Quantum Electrodynamics, Advanced Book Classics (Westview Press, Boulder, 1998).

[30] L. L. Foldy and S. A. Wouthuysen, Phys. Rev. 78, 29 (1950)

[31] E. de Vries, Fortschr. Phys. 18, 149 (1970).

[32] J. P. Costella and B. H. J. McKellar, Amer. J. Phys. 63, 1119 (1995)

[33] S. S. Schweber, An introduction to relativistic quantum field theory (Dover, Mineola, 2005).

[34] P. Caban, J. Rembieliński, and M. Włodarczyk, Ann. Phys. (N. Y.) 330, 263 (2013).

[35] M. Czachor, Phys. Rev. A 55, 72 (1997).

[36] J. Hilgevoord and S. A. Wouthuysen, Nucl. Phys. 40, 1 (1963).

[37] A. S. Wightman, in Relations de dispersion et particules élémentaires (Herman, Paris, 1960) Chap. V.

[38] V. Bargmann, L. Michel, and V. L. Telegdi, Phys. Rev. Lett. 2, 435 (1959)

[39] A. Chakrabarti, J. Math. Phys. 4, 1215 (1963)

[40] F. Gürsey, in High energy physics (Gordon and Breach, New York, 1965) pp. 53-88.

[41] F. Gürsey, Phys. Lett. 14, 330 (1965)

[42] T. Choi, J. Korean Phys. Soc. 62, 1085 (2013)

[43] M. H. L. Pryce, Proc. R. Soc. London, Ser. A 150, 166 (1935)

[44] M. H. L. Pryce, Proc. R. Soc. London, Ser. A 195, 62 (1948)

[45] A. J. Macfarlane, J. Math. Phys. 4, 490 (1963)

[46] R. A. Berg, J. Math. Phys. 6, 34

[47] L. H. Ryder, General Relativity and Gravitation 31, 775 (1999)

[48] D. M. Fradkin and R. H. Good, Jr., Rev. Mod. Phys. 33, 343 (1961)

[49] I. Kirsch, L. H. Ryder, and F. W. Hehl, "The Gordon decompositions of the inertial currents of the Dirac electron correspond to a Foldy-Wouthuysen transformation," (2001), arXiv:hep-th/0102102, arXiv:hep-th/0102102

[50] J. J. Sakurai and J. Napolitano, Modern Quantum Mechanics, 2nd ed. (Pearson Education, Upper Saddle River, 2010).

[51] P. Caban, J. Rembieliński, and M. Włodarczyk, Phys. Rev. A 88, 022119 (2013)

[52] T. Stöhlker, P. Mokler, K. Beckert, F. Bosch, H. Eickhoff, B. Franzke, M. Jung, Y. Kandler, O. Klepper, C. Kozhuharov, R. Moshammer, F. Nolden, H. Reich, P. Rymuza, P. Spädtke, and M. Steck, Phys. Rev. Lett. 71, 2184 (1993)

[53] D. Robbins, P. Beiersdorfer, A. Faenov, T. Pikuz, D. Thorn, H. Chen, K. Reed, A. Smith, K. Boyce, G. Brown, R. Kelley, C. Kilbourne, , and F. Porter, Phys. Rev. A 74, 022713 (2006) 
[54] H.-J. Kluge, T. Beier, K. Blaum, L. Dahl, S. Eliseev, F. Herfurth, B. Hofmann, O. Kester, S. K. ki, C. Kozhuharov, G. Maero, W. Nörtershäuser, J. Pfister, W. Quint, U. Ratzinger, A. Schempp, R. Schuch, T. Stöhlker, R. C. Thompson, M. Vogel, G. Vorobjev, D. F. A. Winters, and G. Werth, in Current Trends in Atomic Physics Advances in Quantum Chemistry, Vol. 53, edited by S. Salomonson and E. Lindroth (Academic Press, 2008).

[55] H. A. Bethe and E. E. Salpeter, Quantum Mechanics of One- and Two-Electron Atoms, Dover Books on Physics (Dover, Mineola, 2008).

[56] B. Thaller, Advanced Visual Quantum Mechanics (Springer, Heidelberg, 2000).

[57] J. D. Bjorken and S. D. Drell, Relativistic quantum mechanics, International series in pure and applied physics (McGraw-Hill, New York, 1964).

[58] Note that due to a typo the explicit expression for the Fouldy-Wouthuysen mean position operator given in the publication by Fouldy and Wouthuysen is wrong; an error that has propagated through many publications [31, 61].

[59] D. M. Wolkow, Z. Phys. 94, 250 (1935)

[60] J. Fröhlich and U. Studer, Rev. Mod. Phys. 65, 733 (1993).

[61] J. R. Ellis and G. Siopsis, J. Phys. A: Math. Gen. 15, L259 (1982) 